University of Wollongong

Research Online

SMART Infrastructure Facility - Papers

Faculty of Engineering and Information

Sciences

2017

\title{
Hybrid method for building extraction in vegetation-rich urban areas from very high-resolution satellite imagery
}

\author{
Ajith Jayasekare \\ University of Wollongong, asj968@uowmail.edu.au \\ Rohan Wickramasuriya \\ University of Wollongong, rohan@uow.edu.au \\ Mohammad-Reza Namazi-Rad \\ University of Wollongong,mrad@uow.edu.au \\ Pascal Perez \\ University of Wollongong, pascal@uow.edu.au \\ Gaurav Singh \\ Commonwealth Scientific Industrial Research Organisation
}

Follow this and additional works at: https://ro.uow.edu.au/smartpapers

Part of the Engineering Commons, and the Physical Sciences and Mathematics Commons 


\title{
Hybrid method for building extraction in vegetation-rich urban areas from very high-resolution satellite imagery
}

\begin{abstract}
A continuous update of building information is necessary in today's urban planning. Digital images acquired by remote sensing platforms at appropriate spatial and temporal resolutions provide an excellent data source to achieve this. In particular, high-resolution satellite images are often used to retrieve objects such as rooftops using feature extraction. However, high-resolution images acquired over built-up areas are associated with noises such as shadows that reduce the accuracy of feature extraction. Feature extraction heavily relies on the reflectance purity of objects, which is difficult to perfect in complex urban landscapes. An attempt was made to increase the reflectance purity of building rooftops affected by shadows. In addition to the multispectral (MS) image, derivatives thereof namely, normalized difference vegetation index and principle component (PC) images were incorporated in generating the probability image. This hybrid probability image generation ensured that the effect of shadows on rooftop extraction, particularly on light-colored roofs, is largely eliminated. The PC image was also used for image segmentation, which further increased the accuracy compared to segmentation performed on an MS image. Results show that the presented method can achieve higher rooftop extraction accuracy $(70.4 \%)$ in vegetation-rich urban areas compared to traditional methods.

\section{Disciplines}

Engineering | Physical Sciences and Mathematics

\section{Publication Details}

Jayasekare, A. S., Wickramasuriya, R., Namazi-Rad, M., Perez, P. \& Singh, G. (2017). Hybrid method for building extraction in vegetation-rich urban areas from very high-resolution satellite imagery. Journal of Applied Remote Sensing, 11 (3), 036017-1-036017-12.
\end{abstract}




\title{
Hybrid method for building extraction in vegetation-rich urban areas from very high-resolution satellite imagery
}

\author{
Ajith S. Jayasekare, ${ }^{\mathrm{a}, *}$ Rohan Wickramasuriya, ${ }^{\mathrm{a}}$ \\ Mohammad-Reza Namazi-Rad, ${ }^{\text {b }}$ Pascal Perez, ${ }^{a}$ and Gaurav Singh ${ }^{\mathrm{c}}$ \\ ${ }^{a}$ University of Wollongong, SMART Infrastructure Facility, Wollongong, \\ New South Wales, Australia \\ ${ }^{b}$ University of Wollongong, National Institute for Applied Statistics Research Australia, \\ Wollongong, New South Wales, Australia \\ ${ }^{c}$ CSIRO, Digital Productivity and Services Flagship, Clayton, Victoria, Australia
}

\begin{abstract}
A continuous update of building information is necessary in today's urban planning. Digital images acquired by remote sensing platforms at appropriate spatial and temporal resolutions provide an excellent data source to achieve this. In particular, high-resolution satellite images are often used to retrieve objects such as rooftops using feature extraction. However, high-resolution images acquired over built-up areas are associated with noises such as shadows that reduce the accuracy of feature extraction. Feature extraction heavily relies on the reflectance purity of objects, which is difficult to perfect in complex urban landscapes. An attempt was made to increase the reflectance purity of building rooftops affected by shadows. In addition to the multispectral (MS) image, derivatives thereof namely, normalized difference vegetation index and principle component (PC) images were incorporated in generating the probability image. This hybrid probability image generation ensured that the effect of shadows on rooftop extraction, particularly on light-colored roofs, is largely eliminated. The PC image was also used for image segmentation, which further increased the accuracy compared to segmentation performed on an MS image. Results show that the presented method can achieve higher rooftop extraction accuracy $(70.4 \%)$ in vegetation-rich urban areas compared to traditional methods. ( $) 2017$ Society of Photo-Optical Instrumentation Engineers (SPIE) [DOI: 10.1117/1.JRS.11.036017]
\end{abstract}

Keywords: rooftop extraction; feature extraction; urban landscapes; probability image; remote sensing.

Paper 170133 received Feb. 14, 2017; accepted for publication Jul. 28, 2017; published online Aug. 23, 2017.

\section{Introduction}

Expansion of urban areas is a globally observed phenomenon that changes the urban environment by adding new structures or by altering existing structures. As a result, a continuous update of buildings and their properties is important for various planning activities, such as transport, environmental, and hazard management. Some properties of buildings show clear relationship with other properties, such as the one observed between the rooftop and the footprint of a building. Hence, rooftop provides an excellent proxy for a building's footprint. Given the rooftops are clearly visible in high-resolution (HR) remotely sensed images and such data are ubiquitous now, satellite images are commonly used for rooftop identification ${ }^{1,2}$ using "feature extraction" techniques.

Feature extraction is a dimension reduction technique. Image-based feature extraction was introduced in the early 60s' for character recognition. ${ }^{3,4}$ In the late 1980 s, feature extraction techniques were used for the first time in spatial sciences with digital images as the data source. ${ }^{5}$ During the last two decades, this technique has been used in different fields related to spatial sciences. For example, Jahjah and Ulivieri ${ }^{6}$ used feature extraction in an archaeological

*Address all correspondence to: Ajith S. Jayasekare, E-mail: asj968@uowmail.edu.au

$1931-3195 / 2017 / \$ 25.00$ (C) 2017 SPIE 
study. The extraction was based on the archaeological structures, and feature extraction was used to analyze the structure and forms in the archaeological site. Sebari and $\mathrm{He}^{7}$ used feature analysis technique for urban land use analysis and urban object identification. Urban object identification is a complex process as the urban environment is characterized by a wide range of size, ${ }^{8}$ shape,${ }^{1,2}$ and texture variations in objects.

HR images acquired over urban environments contain a significant amount of noise caused by shadows and vegetation, thus posing a challenge to rooftop extraction. ${ }^{9}{ }^{10}$ Shadows increase the error of omission. ${ }^{9}$ This challenge is exacerbated by the modern urban planning practices, in particular the emphasis on preserving every square meter of natural area possible, that increase the diversity in the urban landscape. ${ }^{11}$ While urban landscapes enjoy great benefits brought by the increased presence of natural elements, the information collection mechanism that we discuss in this paper, which is feature extraction, needs to be supported by innovative methods that enhance the purity of the reflectance associated with the urban objects of interest from such landscapes.

The main objective of this study is to develop a method to detect building rooftops in vegetation-rich urban areas using HR/very high-resolution (VHR) satellite imagery. Depending on solar zenith and azimuth angles, surrounding large vegetation cast shadows on rooftops. These shadows alter the typical reflectance pattern of the rooftop. Also, large trees that partially obstruct the view affect the accuracy of rooftop area estimation. Hence, we place a particular emphasis on minimizing the interference of large trees in the form of shadows and the partial obstruction of view. We describe a method that reduces the effect of shadows on the rooftop extraction process yielding higher feature extraction accuracy.

The remainder of this article is organized as follows. We first provide a basic background and theory related to the methods used in this paper. The second section discusses the method and related literature associate with each step. The third section is dedicated to result and discussion. Finally, we draw conclusions based on the observed results.

\section{Background}

Spatial resolution is a key property of satellite images, and the pixel size is the measuring unit of the spatial resolution. A pixel is measured in millimeters on the image and in meters on the ground. ${ }^{12}$ Based on the pixel size, satellite images are categorized into low spatial resolution, moderate spatial resolution, HR, and VHR. VHR images, the data type used in this study, are those having spatial resolutions better than $1 \mathrm{~m} .{ }^{13}$ While there are a number of satellites capturing VHR images including IKONOS, QuickBird, and SPOT, our imagery data come from WorldView2. The WorldView2 sensors capture multispectral (MS) images at $2 \mathrm{~m}$ and panchromatic images at $0.5-\mathrm{m}$ spatial resolutions. This is the data source used by Souza dos Anjos et al. ${ }^{14}$ in their urban feature extraction study.

Feature extraction from satellite images usually starts with image classification that is divided into two types: pixel-based and object-based. Pixel-based image classification uses the spectral information to classify the image, but it fails to use spatial and textural information. ${ }^{15}$ Textural and spatial variations captured by VHR images are vital sources of information for object separation in complex landscapes such as urban areas. Object-based image analysis (OBIA) has been used to overcome most of the problems associated with pixel-based classifications. For example, in segmentation, an image is split into homogeneous groups based on both the spectral and the spatial uniformity. ${ }^{16}$

Within a single class of interest (e.g., roof), one can usually identify several subclasses (e.g., different roofing materials) that need to be accounted for. Some research focuses only on a single class such as buildings ${ }^{1}$ where specific properties related to the ground objects, such as sharp spatial and textural variations, are used to demarcate object boundaries.

Depending on the azimuth angle at the time of image acquisition, shadows created by ground objects can be a nuisance. Liu and Prinet ${ }^{17}$ had used a feature and area-based approach to identify different regions in shadow-affected images. They used a probability function to separate the buildings from the rest. Femiani et al. ${ }^{18}$ used shadows as an advantage in extracting rooftops from aerial images. The main drawback of this method is that it is not possible to extract rooftop areas under the shadow. Extraction of the rooftop covered by shadows is considered in our study to offer a better solution to rooftop extraction in vegetation-rich urban areas. 


\section{Methods}

\subsection{Image Analysis}

\subsubsection{Satellite images and image enhancement techniques}

Spatial resolution of an image is positively correlated with the embedded information. ${ }^{19}$ In HR and VHR images, usually several pixels form a single object compared to low-resolution images where one pixel may contain two or more different objects. Thus, very high spatial resolution, as observed in WorldView2 panchromatic band, allows us to take the path of OBIA. Equally important in the process of isolating objects from imagery is the spectral signatures of the objects, which are the unique reflectance of electromagnetic energy caused by the ground objects as recorded in MS bands. Interband arithmetic operations applied to MS images can amplify weak and hidden signatures. It is also possible to combine high spatial resolution of panchromatic images and high spectral resolution of MS images using a process called image fusion or pan-sharpening.

Pan-sharpening is an image processing technique used to merge images of two spatial resolutions, typically HR panchromatic and low-resolution MS while preserving the spectral information. ${ }^{20}$ Pan-sharpening outputs an image of which spatial resolution is similar to a panchromatic image while carrying all the spectral bands present in the MS image. Different methods are available for spatial enhancement through pan-sharpening [e.g., intensity-huesaturation $^{21}$ and principal component analysis (PCA)].

In this study, subtractive resolution merge (SRM) ${ }^{22}$ was used as an image enhancement technique. The WorldView2 MS images with 2-m spatial resolutions were used as low-resolution images and panchromatic images with $0.5 \mathrm{~m}$ were used as HR images. The SRM is capable of controlling both spectral and spatial retentions. ${ }^{23}$ A limitation of the SRM is that the algorithm can apply only for the dual resolution sensors where the spatial resolution ratio is $1: 4 .^{24}$ WorldView2 images used in our study fulfill this requirement. The SRM algorithm also reduces the boundary error caused by spatial mixing. ${ }^{25}$ The resultant image has a $0.5-\mathrm{m}$ spatial resolution with four spectral bands [red, green, blue, and near-infrared (NIR)].

\subsubsection{Normalized difference vegetation index}

Normalized difference vegetation index (NDVI) is an arithmetic calculation between red (R) and NIR bands $(\mathrm{NDVI}=\mathrm{NIR}-\mathrm{R} / \mathrm{NIR}+\mathrm{R}) \cdot{ }^{26}$ Vegetation reflects most of the visible and NIR wavelengths depending on the healthiness, ${ }^{27}$ internal or external structure, age, water status, and mineral ${ }^{28}$ stresses of the vegetation. Accordingly, higher NDVI values are associated with the vegetation areas whereas the lower values represent other objects, such as buildings, bare soil, and water bodies. One explanation for this is that chlorophyll absorbs red wavelength for photosynthesis and reflects almost all NIR, thus keeping NDVI values higher for the vegetation. $^{29}$ This helps to delineate the vegetated area in an image.

\subsubsection{Dimensionality reduction}

Image datasets usually consist of redundant data. Dimensionality reduction methods are used to remove those redundant information (minimum noise fraction transformation, PCA, and singular value decomposition (SVD)]. The PCA and SVD show similarity and effectively used for the white noise reduction. ${ }^{30,31}$ These dimensionality reduction techniques facilitate many algorithms to perform under low dimensional and uncorrelated datasets well. ${ }^{32}$

Principal component (PC) analysis is a statistical procedure that converts the original correlated variables into linearly uncorrelated variables or PCs. In image analysis, it is an image compression technique that preserves the available variation present in the source data. ${ }^{33} \mathrm{~A}$ single feature in an HR satellite image usually consists of several pixel values, and PCA reduces the complexity of such information. In other words, PCA preserves the pattern in the image while emphasizing the similarities and the differences. ${ }^{34}$ In our study, the image comprising the first three principal components was used as an input to the probability image calculation. 


\subsection{Constructing the Probability Image}

Probability images are generated using input images and training samples. Choice of input could vary depending on the type of study. For example, different band combinations and a PC image were used to extract swimming pools in a study conducted in Ref. 35. The inputs used to generate the probability image in our study include pan-sharpened MS image (red, green, blue, and NIR), PC image, and NDVI image, which were derived using foregoing techniques. Input PC image ensures that the interobject variation is maintained despite the low dimensionality of the image. $^{33}$

Vegetation is a prominent land used in the urban landscape that acts as noise in the ground object extraction process. ${ }^{36}$ NDVI is widely used in vegetation analysis ${ }^{37}$ and has been used to distinguish vegetation from other objects. ${ }^{10}$ The NDVI image helps assign lower probability values for vegetation and higher values for nonvegetative objects, such as roofs and roads.

To feed the probability image calculation, we collected a building rooftop sample that represents the diversity in terms of color, texture, and shapes. In addition to the buildings, training samples were collected to cover other objects, such as building shadows and water bodies. Using these samples, original images, and derivatives thereof, two probability images were generated. One probability image was generated using all three input images (MS, PC, and NDVI), and the second image was generated using two input images (MS image and NDVI image). Furthermore, the probability image for roads was generated separately to eliminate any confusion with the black color rooftops.

\subsection{Image Segmentation and Segment Selection}

Image segmentation is a process by which uniform areas in an image are identified based on criteria, such as texture, color, and smoothness. ${ }^{38}$ The process consists of several subprocesses namely, build, modify, grow, merge, cut, or shrink objects. ${ }^{39}$ There are several approaches available for image segmentation. Most common image segmentation methods are thresholding, edge detection, histogram-based method, and region growing. Apart from those methods, full lambdaschedule (FLS) algorithm performs segmentation based on spatial and spectral information. ${ }^{40}$ The algorithm takes each pixel as a separate region to begin with. Then, adjacent segments are merged iteratively based on a combination of spectral and spatial information. ${ }^{41}$ Furthermore, the FLS segmentation is a stepwise procedure that goes through a sequence of algorithms. It first uses the algorithm introduced in Ref. 42 to calculate the $\lambda$ values. The second algorithm performs the merging of adjacent regions $(i, j)$ when the cost, $t_{i, j}$ is less than or equal to a threshold lambda value ( $\lambda$ stop).

$$
t_{i j}=\frac{\frac{\left|O_{i}\right| \times\left|O_{j}\right|}{\left|O_{i}\right|+\left|O_{j}\right|}\left\|u_{i}-u_{j}\right\|^{2}}{\text { length }\left[\partial\left(O_{i}, O_{j}\right)\right]},
$$

where $O_{i}$ and $O_{j}$ represent two segments, and $u_{i}$ and $u_{j}$ identify the average values of the particular regions. The Euclidean distance between spectral values of regions $(i, j)$ is represented by $\left\|u_{i}-u_{j}\right\|^{2}$. The denominator represents the length of the common boundary between region $i$ and $j$.

The segmentation process requires a base image as its main input. We used two different base images in our analysis namely, the original MS image and the PC image. In segmenting an image, different weights can be assigned to spectral and spatial components. Ryherd and Woodcock ${ }^{43}$ stated that the accuracy of segmentation improves when both the spectral and the textural properties are used. The best weight combination for the segmentation was achieved using a trial-and-error method. The segmentation was performed using ERDAS Imagine Objective software where it provides a graphical user interface to change the parameters. Table 1 shows the weight combinations assigned to spectral and textural components while shape and size weights were kept at 0.01 . Additionally, pixel ratio for the segmentation was kept constant as 50, with 10 and 150 margins. Final results not only depend on the segmentation but also the process used for probability image segment selection. 
Table 1 Weights used in the image segmentation.

\begin{tabular}{|c|c|c|c|c|c|c|c|c|}
\hline \multirow[b]{3}{*}{ Property } & \multicolumn{4}{|c|}{ Probability image from MS, PC, and NDVI } & \multicolumn{4}{|c|}{ Probability image from MS and NDVI } \\
\hline & \multicolumn{2}{|c|}{$\begin{array}{l}\text { Segmentation } \\
\text { based on } \\
\text { PC image }\end{array}$} & \multicolumn{2}{|c|}{$\begin{array}{l}\text { Segmentation } \\
\text { based on } \\
\text { MS image }\end{array}$} & \multicolumn{2}{|c|}{$\begin{array}{l}\text { Segmentation } \\
\text { based on } \\
\text { PC image }\end{array}$} & \multicolumn{2}{|c|}{$\begin{array}{l}\text { Segmentation } \\
\text { based on } \\
\text { MS image }\end{array}$} \\
\hline & $\begin{array}{c}\text { High } \\
\text { weight on } \\
\text { spectral }\end{array}$ & $\begin{array}{l}\text { High } \\
\text { weight on } \\
\text { texture }\end{array}$ & $\begin{array}{l}\text { High } \\
\text { weight on } \\
\text { spectral }\end{array}$ & $\begin{array}{l}\text { High } \\
\text { weight on } \\
\text { texture }\end{array}$ & $\begin{array}{c}\text { High } \\
\text { weight on } \\
\text { spectral }\end{array}$ & $\begin{array}{l}\text { High } \\
\text { weight on } \\
\text { texture }\end{array}$ & $\begin{array}{c}\text { High } \\
\text { weight on } \\
\text { spectral }\end{array}$ & $\begin{array}{l}\text { High } \\
\text { weight on } \\
\text { texture }\end{array}$ \\
\hline Spectral & 0.61 & 0.50 & 0.90 & 0.40 & 0.60 & 0.50 & 0.90 & 0.40 \\
\hline Texture & 0.10 & 0.75 & 0.70 & 0.80 & 0.10 & 0.75 & 0.70 & 0.80 \\
\hline
\end{tabular}

\subsection{Accuracy Assessment}

The accuracy of the rooftop detection from satellite images is commonly measured using false detection and true detection. ${ }^{2}$ Calculating error matrix is one of the most commonly used techniques to identify the accuracy. ${ }^{29}$ Building area accuracy in our study can be considered as the user accuracy in error matrix terms. False detection accuracy is concerned with the other objects that are falsely detected as rooftops while true detection expresses the percentage of correctly detected rooftops out of the total number of rooftops. While these two statistics focus on the numbers, we have used two other statistics to establish the accuracy in terms of area of the extracted features. The accuracy assessment is based on randomly selected samples from study areas.

The extracted feature should ideally cover the complete area of a building. If it is less than the manually extracted building area, it is then considered an error in rooftop extraction. The accuracy of the building area is calculated as a percentage [Eq. (2)]. The manual building extraction process is based on the pan-sharpened image $(0.5-\mathrm{m}$ resolution), and the manual digitizing is performed to demarcate the building extent.

$$
\begin{gathered}
\text { Building area accuracy }=\frac{\text { Correctly extracted feature area within the sample areas }}{\text { Manually extracted building area from pan sharpened }} \times 100 . \\
\text { MS image within the sample areas }
\end{gathered}
$$

Feature extraction targets specific objects. If other objects are falsely extracted as target features, it is considered an error that results in overestimation. False extraction (area) is calculated based on the sample area, and the building area is subtracted from the sample area to get the "total area outside the buildings"

False extraction(area)

$$
=\frac{\text { Extracted feature area outside the buildings and within sample areas }}{\text { Total area outside the building and within the sample areas }} \times 100 \text {. }
$$

\subsection{Tree Canopy and Shadows}

Leaf area index (LAI) is a key factor that describes the radiation interaction ${ }^{44}$ of the tree canopy. Numerous researches have shown that NDVI can be used to estimate LAI, which in turn explains the canopy density. We used the relationship stated in Ref. 45 to estimate the leaf density in deferent sections of a tree canopy. An unsupervised classification of the NDVI image was used to differentiate the high and low dense area of the canopy. 


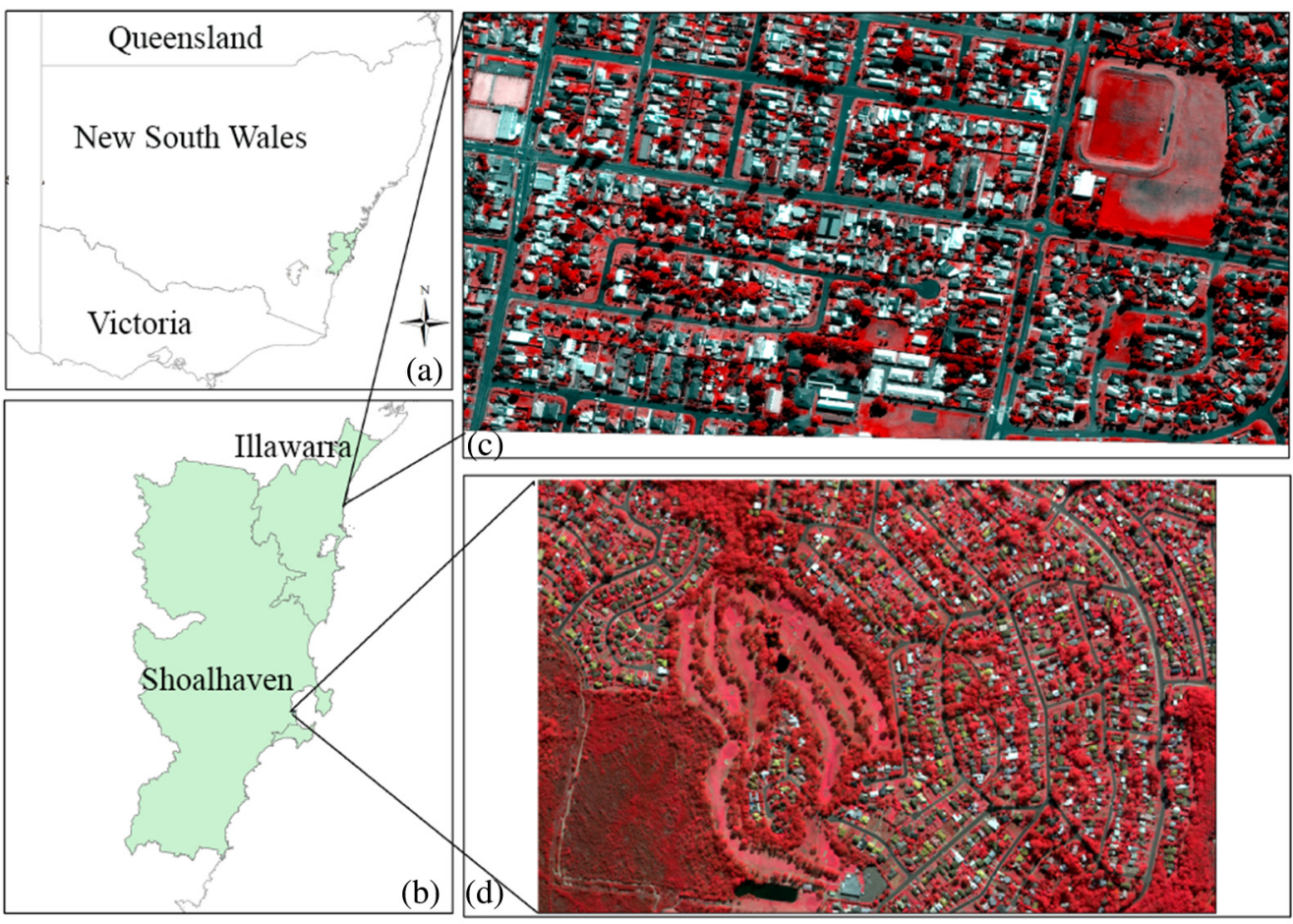

Fig. 1 (a) Study area in NSW, (b) study area within south coast of NSW, (c, d) two study areas in false color composite [WorldView2 satellite image (blue-layer 2, green-layer 3, and red-layer 4)].

\subsection{Study Area}

The study was conducted for two areas in the south coast of New South Wales state (Fig. 1). The area is predominantly occupied by medium to low density urban residential land use. Large trees that partially block the building rooftops as well as shadows of such trees that fall on rooftops are abundant in the study area. Hence, this area presents itself as an ideal test bed to experiment our hybrid rooftop extraction procedure.

\section{Results and Discussion}

Two different combinations of inputs were used to generate the probability images. One probability image was generated using all three input images (MS, NDVI, and PC), whereas the second probability image was generated using only MS and NDVI images. Figure 2 shows the generated probability images. Both of these probability images show high probability values for buildings and roads. However, the PC integrated image makes it easy to distinguish building rooftops from adjacent ancillary features, such as shadows and driveways, as seen in Figs. 2(e) and 2(f). The subsequent segmentation process used these probability images as input.

Figure 3 shows a sample of probability variation for light-colored rooftops affected by shadows. The probability image generated based on all three inputs [Fig. 3(b)] assigns similar probability values for the entire rooftop area irrespective of the fact that the rooftop is partially covered by shadows. The second probability image that was not provided with the PC image as one of the inputs assigns lower probability values for areas affected by shadow [Fig. 3(c)]. Furthermore, PC-included probability image has successfully isolated the rooftop from the background, whereas the other probability image failed to do so.

Shadows of trees are a dominant interfering factor to the rooftop extraction process. ${ }^{17}$ Intensity of the observed shadows varies depending on the canopy density. The methodology used in this study provides the best results under low intensity shadows. For example, Fig. 4(a) shows a rooftop with shadow that is created by a low dense canopy, and Fig. 4(b) shows the extracted rooftop in this instance. 


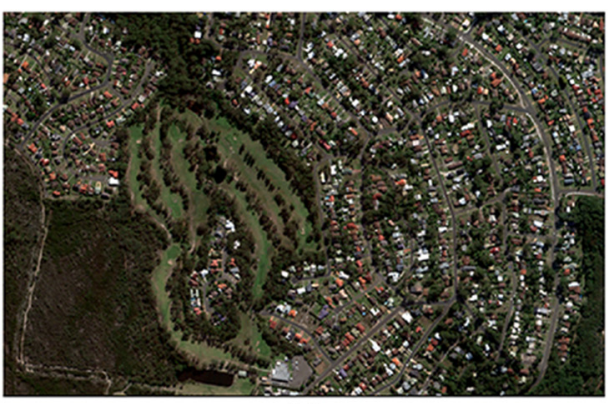

(a)

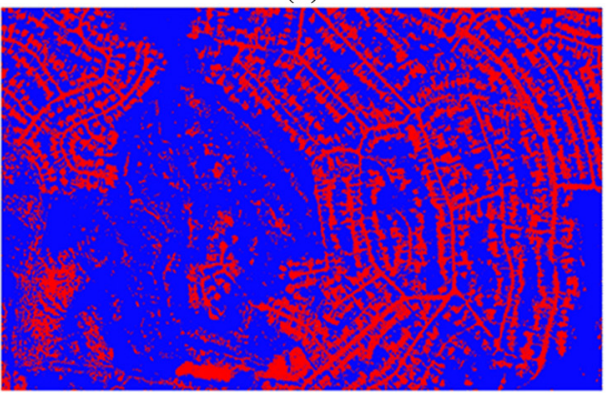

(c)

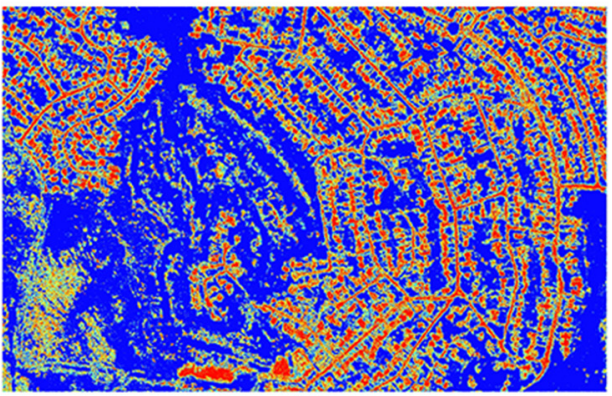

(e)

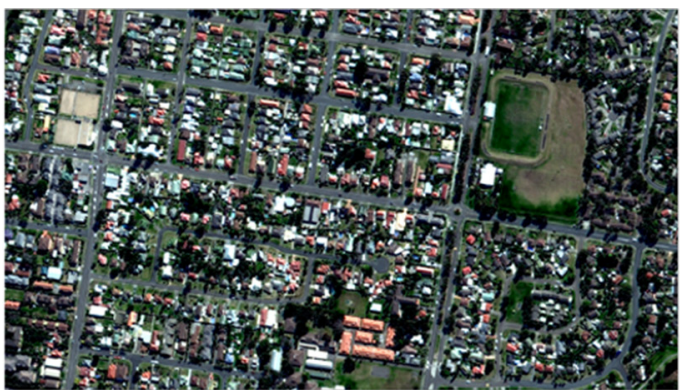

(b)

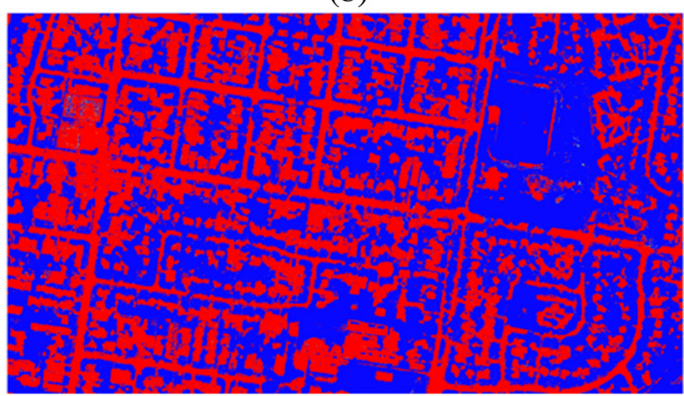

(d)

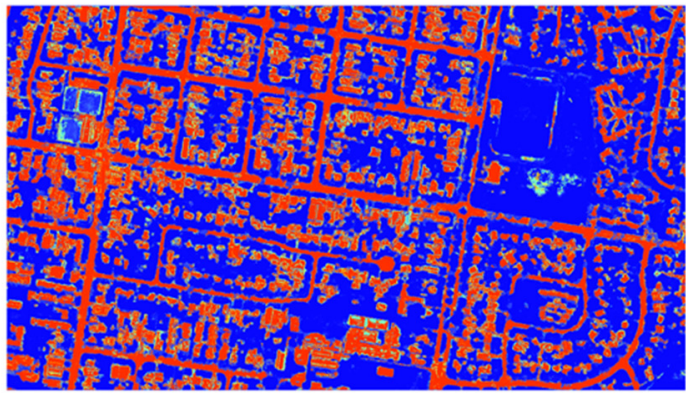

(f)

0 $0.25 \quad 0.5 \mathrm{Km}$

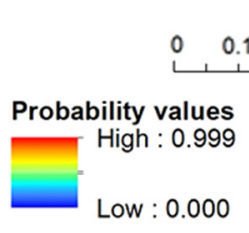

Fig. 2 Generated images: (a) true color composite of site 1, (b) true color composite of site 2, (c, d) probability images generated for two sites based on MS and NDVI image inputs only, $(e, f)$ probability images generated for two sites based on MS, NDVI, and PC image inputs.

In segmentation, it is important to segment the image from object boundaries such as rooftops from ground, rooftop from shadows, etc. If not it increases the error of commission by extracting nonbuilding area as building. The selected weights segment most of the roof boundaries. However, it also segments a roof into different segments. Created probability image creates the high probability area within the roof, and it increases the roof segmentation selections that belong to a roof. As a result, error of omission is reduced from the final result.

More critical, however, is the tonal contrast between the rooftop and the shadow. For example, two rooftops are shown in Figs. 4(a) and 4(d), where Fig. 4(a) shows a light-colored rooftop, whereas Fig. 4(d) depicts a darker-colored rooftop. Both these rooftops are affected by shadows caused by canopies of similar thickness. Extracted rooftops are shown in Figs. 4(b) and 4(e). The extracted rooftop for the dark-colored rooftop is incomplete, although both canopies show similar NDVI variation [Figs. 4(c) and 4(f)] indicating similar shadow characteristics.

To put this study into perspective, it is worth looking at results achieved in similar studies elsewhere. For example, Taubenbock et al. ${ }^{46}$ used MS and NDVI images for rooftop extraction. As we show in Fig. 5, it is clear that a procedure involving MS and NDVI images fails to remove 


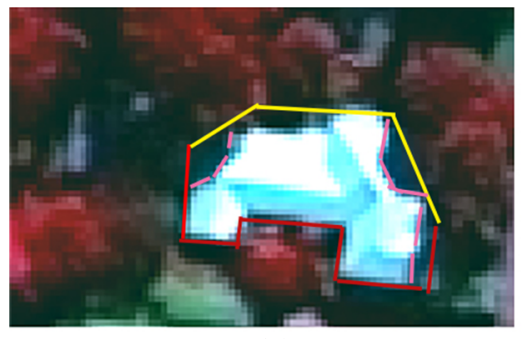

(a)

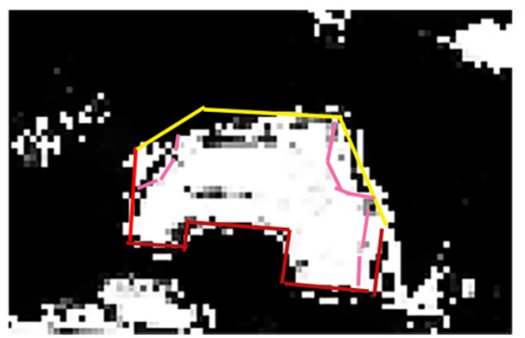

(b)
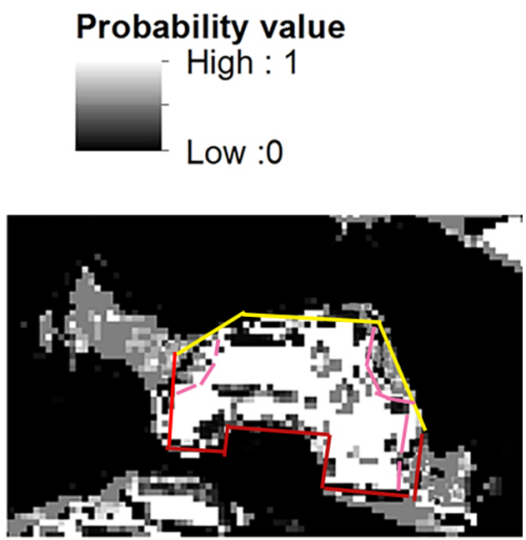

(c)

Fig. 3 Probability value variation on light-colored rooftop. (a) Rooftop in standard false color composite, (b) probability value from all three images (MS, PC, and NDVI), and (c) probability value from MS and NDVI images.

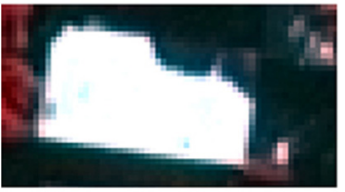

(a)

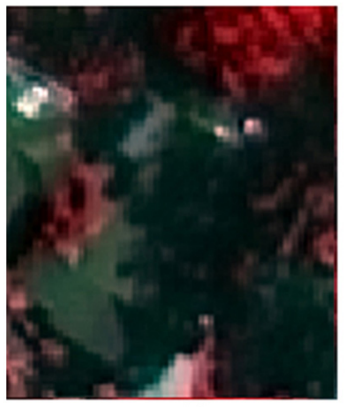

(d)

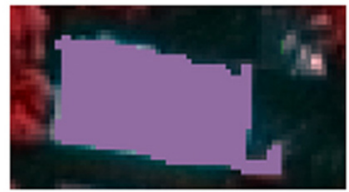

(b)

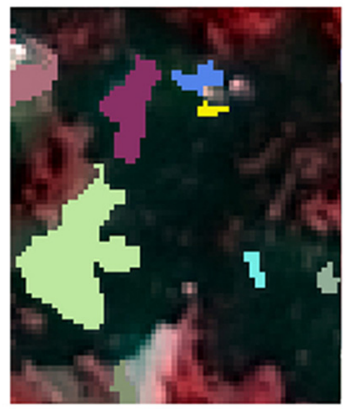

(e)

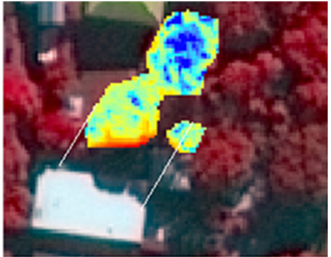

(c)

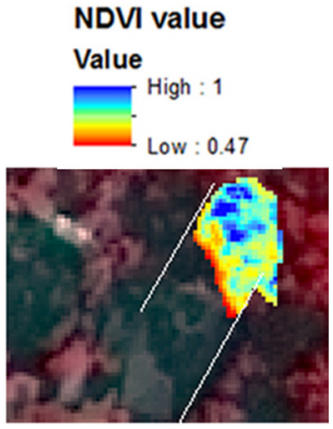

(f)

Fig. 4 Rooftop extraction under different textural contrast (a) a light-colored roof with canopy shadow, (b) extracted rooftop for light-colored roof, (c) NDVI variation of the vegetation that casts a shadow on a light-colored roof, (d) dark rooftop with canopy shadow, (e) extracted rooftop fragments, and (f) NDVI variation of the vegetation that casts a shadow on a dark-colored roof.

the effect of shadow and results in a partial extraction of the rooftop. When PC image is included together with MS and NDVI images, the extraction process yields a more complete demarcation of the rooftop by eliminating the effect of shadow [Fig. 5(c)].

Overall accuracy of the rooftop extraction process is significantly influenced by the weights assigned to spectral and textural components in the segmentation step (Table 1). Accuracy also depends on the base image on which segmentation is performed. We used MS image and PC image separately as the base image. Figure 6 shows the overall accuracy achieved based on different combinations of base image, spectral, and textural weights. The highest average 


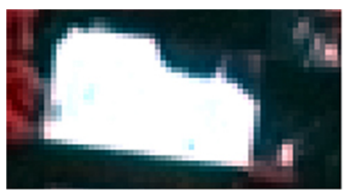

(a)

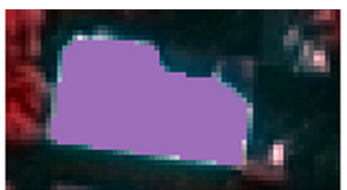

(b)

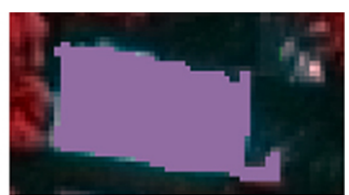

(c)

Fig. 5 Rooftop extraction using different combinations of input images. (a) Observed rooftop with shadow, (b) extracted rooftop area using MS and NDVI images, and (c) extracted rooftop area using MS, NDVI, and PC images.

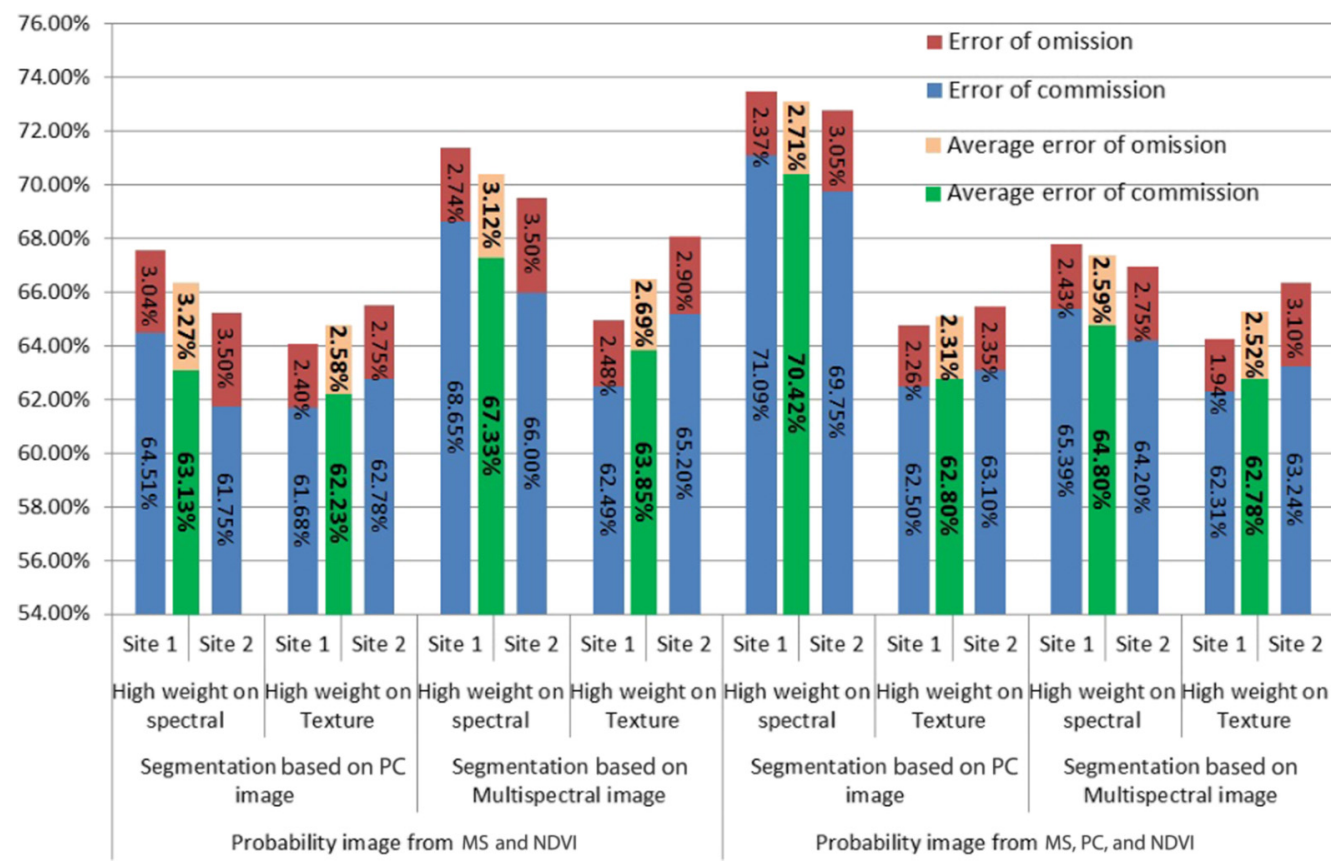

Fig. 6 Feature extraction accuracy based on different combinations of base image, probability image, and weights for spectral and textural components.

accuracy from both sites of $70.42 \%$ is achieved when the probability image is generated using MS, NDVI, and PC images, base image is PCA, and the spectral weight is high compared to the texture weight (Table 1).

The highest overall accuracy (73.4\%) achieved in a building rooftop extraction study, to the best of our knowledge, is achieved by Xiang et al. ${ }^{2}$ However, their study area was a dense urban area, mostly free from large trees. Accordingly, the rooftop extraction accuracy achieved in this study is the highest for a vegetation-rich urban area.

\section{Conclusions}

Location and properties of buildings serve as vital information in urban planning. Traditionally, digitizing has been used as a method to extract spatial objects such as building rooftops, but it is a time-consuming manual exercise. Most digitizing work is performed using HR satellite imagery, which is an excellent data source for automating the same information extraction process. However, the automated feature extraction process is challenging in vegetation-rich urban areas due to the effect of shadows and partial obstruction of view created by tree canopies.

In this study, we employed a hybrid method that utilizes an MS image, NDVI image, and a PC image to generate the probability image that is a key input in the segmentation process. When this probability image is combined with the same PC image as the base image while assigning a higher weight to spectral component compared to the textural component during segmentation, it yields the highest rooftop extraction accuracy of $70.4 \%$. To the best of our knowledge, this is 
the highest accuracy achieved in a building rooftop extraction study conducted in a vegetationrich urban area using VHR satellite imagery.

Two factors, namely the intensity of the canopy creating the shadow and the tonal contrast between the rooftop and the shadow, greatly governed the rooftop extraction accuracy. Further, the effect of the latter was more pronounce on the accuracy. We envisage that a stepwise procedure that extracts rooftops of similar texture in a single step improves the accuracy significantly. This will be the subject of a future paper.

\section{References}

1. E. Angiati and S. Dellepiane, "Identification of roofs perimeter from aerial and satellite images," in 17th Int. Conf. on Digital Signal Processing (DSP '11) (2011).

2. Y. Xiang, Y. Sun, and C. Li, "A rooftop extraction method using color feature, height map information and road information," Proc. SPIE 8537, 85370T (2012).

3. W. Doyle, "Recognition of sloppy, hand-printed characters," in IRE-AIEE-ACM '60 (Western) Papers Presented at the May 3-5, 1960, Western Joint IRE-AIEE-ACM Computer Conf., pp. 133-142, ACM (1960).

4. R. Bakis, N. M. Herbst, and G. Nagy, "An experimental study of machine recognition of hand-printed numerals," IEEE Trans. Syst. Sci. Cybern. 4(2), 119-132 (1968).

5. R. I. Taniguchi and E. Kawaguchi, "Road network extraction from Landsat TM image," in Third Int. Conf. on Image Processing and Its Applications (1989).

6. M. Jahjah and C. Ulivieri, "Automatic archaeological feature extraction from satellite VHR images," Acta Astronaut. 66(9-10), 1302-1310 (2010).

7. I. Sebari and D.-C. He, "Automatic fuzzy object-based analysis of VHSR images for urban objects extraction," ISPRS J. Photogramm. Remote Sens. 79, 171-184 (2013).

8. J. Leitloff, S. Hinz, and U. Stilla, "Vehicle detection in very high resolution satellite images of city areas," IEEE Trans. Geosci. Remote Sens. 48(7), 2795-2806 (2010).

9. D. A. Aldred and J. Wang, "A method for obtaining and applying classification parameters in object-based urban rooftop extraction from VHR multispectral images," Int. J. Remote Sens. 32(10), 2811-2823 (2011).

10. D. Singh et al., "Building extraction from very high resolution multispectral images using NDVI based segmentation and morphological operators," in Students Conf. on Engineering and Systems (SCES) (2012).

11. J. Humphreys, "Urban planning and vegetation conservation: a case for forward planning," Urban Policy Res. 7(3), 106-116 (1989).

12. E. Chuvieco, Fundamentals of Satellite Remote Sensing, CRC Press, Boca Raton (2010).

13. T. Toutin, "Fine spatial resolution optical sensors," in The SAGE Handbook of Remote Sensing, T. A. Warner, M. D. Nellis, and G. M. Foody, Eds., pp. 108-122, SAGE Publications Ltd., London (2009).

14. C. Souza dos Anjos et al., "Urban feature extraction on simulated WorldView-2 images," in Joint Urban Remote Sensing Event (JURSE '13) (2013).

15. A. Hamedianfar et al., "Improving detailed rule-based feature extraction of urban areas from WorldView-2 image and lidar data," Int. J. Remote Sens. 35(5), 1876-1899 (2014).

16. M. Baatz and A. Schape, "Multiresolution segmentation: an optimization approach for high quality multi-scale image segmentation," in Angewandte geographische Informationsverarbeitung XII: Beiträge zum AGIT-Symp., Wichmann, Salzburg (2000).

17. W. Liu and V. Prinet, "Building detection from high-resolution satellite image using probability model," in Int. Geoscience and Remote Sensing Symp. (2005).

18. J. Femiani et al., "Shadow-based rooftop segmentation in visible band images," IEEE J. Sel. Top. Appl. Earth Obs. Remote Sens. 8(5), 2063-2077 (2015).

19. R. M. Narayanan and M. K. Desetty, "Effect of spatial resolution on information content characterization in remote sensing imagery based on classification accuracy," Int. J. Remote Sens. 23(3), 537-553 (2002).

20. B. Aiazzi et al., "Spatial enhancement of multispectral images on urban areas," in Urban Remote Sensing: Monitoring, Synthesis and Modeling in the Urban Environment, X. Yang, Ed., John Wiley \& Sons, Ltd., Chichester, United Kingdom (2011). 
21. H. Afify, "Intensity-hue-saturation based methods for fusing quickbird images," Int. J. Geoinf. 8(2), 71-79 (2012).

22. S. Y. Zhang et al., "A new method for multi-source remote sensing image fusion," in Proc. IEEE Int. Geoscience and Remote Sensing Symp. (IGARSS '05) (2005).

23. S. Ashraf, L. Brabyn, and B. J. Hicks, "Alternative solutions for determining the spectral band weights for the subtractive resolution merge technique," Int. J. Image Data Fusion 4(2), 105-125 (2013).

24. S. Ashraf, L. Brabyn, and B. J. Hicks, "Introducing contrast and luminance normalisation to improve the quality of subtractive resolution merge technique," Int. J. Image Data Fusion 4(3), 230-251 (2013).

25. X. Yang, "Use of archival Landsat imagery to monitor urban spatial growth," in Urban Remote Sensing: Monitoring, Synthesis and Modeling in the Urban Environment, X. Yang, Ed., pp. 15-33, John Wiley \& Sons, Ltd., Chichester, United Kingdom (2011).

26. J. W. Rouse et al., Eds., "Monitoring vegetation systems in the Great Plains with ERTS," in 3rd ERTS Symp.: National Aeronautics and Space Administration (NASA) (1973).

27. W van Leeuwen, "Visible, near-IR, and shortwave IR spectral characteristics of terrestrial surfaces," in The SAGE Handbook of Remote Sensing, T. A. Warner, M. D. Nellis, and G. M. Foody, Eds., pp. 33-50, SAGE Publications Ltd., London (2009).

28. B. B. Salem, G. L. Andersen, and M. A. Zahran, "Remote sensing and vegetation map of Egypt," in The Vegetation of Egypt, Plant and Vegetation, Vol. 2, pp. 319-333, Springer, Netherlands (2009).

29. J. B. Campbell, Introduction to Remote Sensing, 3rd ed., Guilford Press, New York (2002).

30. S. Danaher et al., "A comparison of the characterisation of agricultural land using singular value decomposition and neural networks," in Neurocomputation in Remote Sensing Data Analysis, pp. 14-27, Springer (1997).

31. M. B. D. Jose and M. P. N. Jose, "Vertex component analysis: a geometric based approach to unmix hyperspectral data-analysis," in Image Processing for Remote Sensing, pp. 149-173, CRC Press (2007).

32. S. Roessner et al., "Potential of hyperspectral remote sensing for analyzing the urban environment," in Urban Remote Sensing: Monitoring, Synthesis and Modeling in the Urban Environment, X. Yang, Ed., John Wiley \& Sons, Ltd., Chichester, United Kingdom (2011).

33. W. Yang et al., "Classification of emerald based on multispectral image and PCA," Proc. SPIE 5637, 684 (2005).

34. I. S. Bajwa and S. I. Hyder, "PCA based image classification of single-layered cloud types," in Proc. of the IEEE Symp. on Emerging Technologies (2005).

35. S. W. Myint and D. Stow, "An object-oriented pattern recognition approach for urban classification," in Urban Remote Sensing: Monitoring, Synthesis and Modeling in the Urban Environment, pp. 129-140, John Wiley \& Sons, Ltd. (2011).

36. E. Tarantino and B. Figorito, "Extracting buildings from true color stereo aerial images using a decision making strategy," Remote Sens. 3(8), 1553-1567 (2011).

37. W. Li, J. D. M. Saphores, and T. W. Gillespie, "A comparison of the economic benefits of urban green spaces estimated with NDVI and with high-resolution land cover data," Landscape and Urban Planning 133, 105-117 (2015).

38. J. Serra, "Image segmentation," in Proc. Int. Conf. on Image Processing (ICIP '03) (2003).

39. M. Baatz, C. Hoffmann, and G. Willhauck, "Progressing from object-based to object-oriented image analysis," in Object-Based Image Analysis, Lecture Notes in Geoinformation and Cartography, T. Blaschke, S. Lang, and G. Hay, Eds., pp. 29-42, Springer, Berlin, Heidelberg (2008).

40. D. J. Robinson, N. J. Redding, and D. J. Crisp, "Implementation of a fast algorithm for segmenting SAR imagery," Technical Report, DSTO-TR-1242, DSTO Electronics and Surveillance Research Laboratory, South Australia (2002).

41. L. Liu et al., "An object-oriented daytime land-fog-detection approach based on the meanshift and full lambda-schedule algorithms using EOS/MODIS data," Int. J. Remote Sens. 32(17), 4769-4785 (2011). 
42. G. Koepfler, C. Lopez, and J.-M. Morel, "A multiscale algorithm for image segmentation by variational method," SIAM J. Numer. Anal. 31(1), 282-299 (1994).

43. S. Ryherd and C. Woodcock, "Combining spectral and texture data in the segmentation of remotely sensed images," Photogramm. Eng. Remote Sens. 62(2), 181-194 (1996).

44. M. T. van Wijk and M. Williams, "Optical instruments for measuring leaf area index in low vegetation: application in arctic ecosystems," Ecol. Appl. 15(4), 1462-1470 (2005).

45. L. Fan et al., "Investigating the relationship between NDVI and LAI in semi-arid grassland in inner Mongolia using in-situ measurements," Theor. Appl. Climatol. 95(1-2), 151-156 (2009).

46. H. Taubenbock et al., "Object-based feature extraction using high spatial resolution satellite data of urban areas," J. Spat. Sci. 55(1), 117-132 (2010).

Ajith S. Jayasekare is a $\mathrm{PhD}$ student at the School of Computer Science, University of Wollongong, Australia. He received his BSc degree in agricultural sciences at the University of Peradeniya, Sri Lanka. Further education is done at the Stuttgart University of Applied Science and followed master's degree in photogrammetry and geoinformatics in 2008 to 2010. After that, he worked for United Nations Development Programme and International Water Management Institute.

Biographies for the other authors are not available. 